\title{
Perfiles de población y uso de los transportes motorizados. Evidencias desde el campus de la Universitat Autònoma de Barcelona (UAB)
}

\author{
Monika Maciejewska \\ Carme Miralles-Guasch \\ Oriol Marquet \\ Universitat Autònoma de Barcelona. Departament de Geografia \\ monika.maciejewska@uab.cat \\ carme.miralles@uab.cat \\ oriol.marquet@uab.cat
}

\section{Resumen}

La ubicación suburbana del campus de la UAB implica que el 94,2\% de la comunidad universitaria usa el transporte motorizado en el desplazamiento cotidiano a su lugar de estudio o trabajo. Al tratarse de unos desplazamientos definidos por un mismo motivo y un único destino, se facilita el análisis de la influencia que el género y la edad ejercen en la utilización de los medios de transporte, especialmente en la elección entre el transporte público y el privado. Este artículo analiza el reparto modal y los principales motivos de elección del modo de transporte de acceso al campus universitario de la UAB. Se encuentran diferencias significativas entre alumnado y personal (docente y administrativo), y también entre hombres y mujeres. Una comprensión profunda del uso de los distintos modos de transporte motorizado con relación a diferentes grupos poblacionales es fundamental para el diseño y la implementación de políticas públicas dirigidas a la reducción de los transportes menos sostenibles.

Palabras clave: commuting; reparto modal; género; edad; movilidad cotidiana 
Resum. Perfils de població $i$ ús dels transports motoritzats. Evidències des del campus de la Universitat Autònoma de Barcelona (UAB)

La ubicació suburbana del campus de la UAB implica que el 94,2\% de la comunitat universitària fa servir el transport motoritzat en el desplaçament quotidià al seu lloc d'estudi o treball. En tractar-se d'uns desplaçaments definits per un mateix motiu i una única destinació, es facilita l'anàlisi de la influència que el gènere i l'edat exerceixen en la utilització dels mitjans de transport, especialment l'elecció entre el transport públic i el privat. Aquest article analitza el repartiment modal i els principals motius d'elecció del mitjà de transport d'accés al campus universitari de la UAB. Es troben evidències significatives entre alumnat $\mathrm{i}$ personal (docent i administratiu), i també entre homes i dones. Una comprensió profunda de l'ús dels diferents mitjans de transport motoritzat amb relació a diferents grups poblacionals és fonamental per al disseny i la implementació de polítiques públiques dirigides a la reducció dels transports menys sostenibles.

Paraules clau: commuting; repartiment modal; gènere; edat; mobilitat quotidiana

Résumé. Profils de population et utilisation des transports motorisés. Recherches sur le campus de l'université Autonome de Barcelone (UAB)

L'emplacement en banlieue du campus de l'UAB signifie que 94,2\% de la communauté universitaire utilise quotidiennement des transports motorisés pour se rendre sur son lieu d'études ou de travail. Ces voyages étant caractérisés par un même motif et une même destination, nous analysons l'influence du sexe et de l'âge sur l'utilisation des moyens de transport, en particulier en ce qui concerne le choix entre les transports publics et privés. Cet article analyse la division modale et les principales raisons de choisir un moyen de transport sur le campus de l'UAB, où il existe des différences significatives entre étudiants et professionnels (enseignants et administrateurs), ainsi qu'entre hommes et femmes. Une compréhension profonde de l'utilisation de différents modes de transport motorisés en fonction des différents groupes de population est fondamentale pour la conception et la mise en œuvre des politiques publiques visant à réduire les transports moins durables.

Mots-clés: commuting; répartition modale; genre; âge; mobilité quotidienne

Abstract. Population profiles and use of motorized transport: Evidence from the Autonomous University of Barcelona (UAB) campus

The suburban location of the Autonomous University of Barcelona (UAB) campus means that $94.2 \%$ of the university community uses motorized transport in their daily travel to their place of study or work. As these trips share the same purpose and a single destination, the influence of gender and age on the use of different modes of transport - especially between public and private transport - can be analyzed in an easier way. This article examines the distribution of and the main reasons for choosing a given mode of transport to the UAB campus. Significant differences were found between students and workers (i.e., teachers and administrators) and between men and women. A deep understanding of the use of different modes of motorized transport in relation to different population groups is fundamental for the design and implementation of public policies aimed at reducing less sustainable transport.

Keywords: commuting; modal split; gender; age; daily mobility 


\section{Sumario}

$\begin{aligned} & \text { 1. Introducción } \text { 4. Género y edad como factores que } \\ & \text { influyen en los desplazamientos en la UAB } \\ & \text { 3. Metodología } \text { 5. Conclusiones } \\ & \text { Referencias bibliográficas }\end{aligned}$

\section{Introducción}

El reparto modal es una de las características que mejor describen el modelo de movilidad de un territorio determinado, por lo que se convierte en uno de los desafíos de las investigaciones sobre movilidad cotidiana. El reto al que se enfrentan las distintas administraciones públicas no deriva de la cantidad de viajes ni de los excesos de la movilidad, definidos por algunos como hipermovilidad (Adams, 1999), sino de los medios de transporte que se utilicen. Los medios privados son los que contribuyen, en mayor medida, a las emisiones de $\mathrm{CO}_{2}$ y al consecuente cambio climático (Woodcock et al., 2007), mientras que los medios activos (caminar y la bicicleta) son los que menos inciden (Lundberg y Weber, 2014). La relación desigual entre los medios de transporte y el medio ambiente hace que el objetivo de las políticas públicas sea la reducción de los viajes en automóvil (Hickman et al., 2013) y no de los desplazamientos en sí mismos.

Los viajes cotidianos, en gran medida, reflejan el dinamismo social y económico de la población, y expresan las actividades cotidianas que realizan los ciudadanos. Es en este contexto donde el análisis de la distribución modal tiene su máximo sentido, pues no solo evidencia qué medios de transporte se utilizan, sino también qué grupos de población realizan los desplazamientos y qué motivos tienen para ello. El colectivo de personas que viajan no es homogéneo. Estas tienen distintas características demográficas, socioeconómicas y culturales, y todas ellas influyen en los medios de transporte utilizados (Pitombo et al., 2011). Además, no hay que menospreciar la influencia - y en algunos casos la exigencia- que ejercen las distintas tipologías de los entornos urbanizados (Næss, 2015).

Con el objetivo de entender el reparto modal de un colectivo poblacional y profundizar en las razones de la elección modal, el presente trabajo analiza los viajes por trabajo o estudio que realizan los miembros de la comunidad universitaria al campus de la Universidad Autónoma de Barcelona (UAB): profesores, personal de servicios y estudiantes. Se trata de una universidad campus, aislada y suburbana, con una amplia oferta de transporte ferroviario y con una importante red viaria de alta capacidad, que genera todos los días más de cien mil desplazamientos, realizados con medios públicos, privados y activos. Es una comunidad que presenta características más homogéneas que otros colectivos de población. Sus miembros tienen en común un viaje generado por el mismo motivo (ir a estudiar o a trabajar) y un único destino (el campus universitario), 
además de tener unos niveles económicos y culturales similares. Esta homogeneidad permite analizar mejor las variables de género y edad con relación al modo de transporte utilizado (Miralles-Guasch et al., 2016).

\section{Marco teórico}

\subsection{Elección y reparto modal}

Rodrigue et al. (2009) definen la distribución modal como la proporción de los viajes realizados en distintos medios de transporte. El reparto modal mide el volumen de medios de transporte utilizados por una comunidad para realizar sus desplazamientos cotidianos en un territorio determinado, y siempre es una medida porcentual entre los medios privados, públicos y activos. Además, al ser los transportes privados los más contaminantes y los que en mayor medida utilizan energías no renovables (Banister, 2011), estos son mucho menos sostenibles que los públicos o los activos (Black, 2010; Schwanen et al., 2011). Este hecho permite utilizar el reparto modal como indicativo de los niveles de sostenibilidad de un lugar determinado.

Aunque buena parte de la literatura académica usa modal split y modal choice como conceptos sinónimos, el primero mide un fenómeno colectivo, relacionado con las características del conjunto de desplazamientos de una comunidad. En cambio, el modal choice hace referencia a una decisión particular (Ory y Mokhtarian, 2009) que, según Witte et al. (2013), expresa un proceso de decisión individual que determina qué medio de transporte se utiliza. Una elección que se realiza antes de cualquier desplazamiento (MirallesGuasch et al., 2014), y que puede ser consciente o formar parte de unos hábitos cotidianos, a menudo repetitivos y automáticos (Garcia-Sierra et al., 2015).

La literatura académica señala que la elección modal depende de las características territoriales y de las peculiaridades socioeconómicas de la población (Banister, 2011; Gutiérrez y García-Palomares, 2007). De este modo, la densidad, el grado de diversidad de usos, los tamaños poblacionales o la tipología y diversidad del espacio público afectan directamente a los transportes utilizados por una comunidad en su movilidad cotidiana (Ewing y Cervero, 2002; Litman, 2012). También el nivel de renta, la edad y el género tienen un papel determinante en el uso de los distintos medios de transporte (Limtanakool et al., 2006; Scheiner, 2010). La influencia de todas estas características en la distribución modal indica que la movilidad no es solo un fenómeno individual, sino que tiene una dimensión colectiva dentro de una diversa estructura social (Ciuffini, 1993) y territorial (Miralles-Guasch et al., 2016).

Sin embargo, en este momento, una de las características más importantes del reparto modal es la relación directa y desigual de los distintos medios de transporte con los impactos ambientales (Schwanen et al., 2011; Tiwari et al., 2011). Unos impactos que se ubican en el nivel de contaminación atmosférica y en la utilización de energías no renovables. Los medios activos (caminar y la bicicleta) y los públicos contaminan y gastan menos energía que los privados. 
Los datos de la UE-27 para 2007 señalan que los coches demandan cuatro veces más energía - por km transportado por pasajero- que el transporte público (ADEME, 2009). El vehículo privado es también el principal responsable de la contaminación atmosférica y acústica. Por tanto, cualquier estrategia para incrementar los niveles de sostenibilidad urbana requiere reducir los impactos que los transportes tienen sobre la atmosfera, lo que implica que un reparto modal más sostenible sea un objetivo prioritario para cualquier Administración (Miralles-Guasch, 2012). De esta forma, y para que las políticas públicas puedan establecer estrategias adecuadas para disminuir los impactos ambientales de los transportes, es necesaria una comprensión profunda de las variables que influyen en la utilización de los distintos medios de transporte (Bhat, 1998).

\subsection{Las características sociodemográficas y el reparto modal. La importancia del género y la edad}

La utilización de los distintos medios de transporte está directamente relacionada con las características sociodemográficas de la población, ya que cada grupo social tiene sus propias necesidades de viaje, vinculadas a sus tipologías de desplazamiento con relación al tiempo de viaje, al recorrido o al modo de transporte (Cerin et al., 2007). Sin embargo, esta relación diferencial de los distintos grupos sociales con el transporte no emerge en los análisis académicos hasta la década de los setenta (Law, 1999). En estos años, se empiezan a reconocer las diferencias existentes en la demanda de transportes. Y esta diversidad afecta a los usos desiguales de los medios de transporte. Aunque este primer reconocimiento se situó en los márgenes del discurso hegemónico durante muchos años, el cambio analítico real no se dio hasta que se sustituyó el sujeto de análisis, que pasó de los transportes (la oferta) a las personas que se desplazaban en distintos modos (la demanda). Con ello, se acuñó el concepto de movilidad como la suma de desplazamientos que hacen las personas (Miralles-Guasch, 2002). Al ser las personas el objeto de análisis, es imprescindible (re)conocer sus características sociodemográficas en relación con sus desplazamientos y el entorno territorial donde se ubican (Garcia-Ramon et al., 2004).

Siguiendo a Witte et al. (2013), el proceso de decisión entre diferentes alternativas de transporte viene determinado por una combinación de factores individuales y colectivos, e influenciado por características sociopsicológicas. Sin embargo, cuando uno de estos grupos de factores es similar para todo el colectivo estudiado, los factores más desiguales sobresalen como variables explicativas (Miralles-Guasch et al., 2016). Así, cuando los factores territoriales y los sociopsicológicos son análogos, los sociodemográficos se pueden estudiar con más profundidad, sin que otros los influyan. En este caso, las características individuales son la edad, el género, la educación, la ocupación, el nivel económico y el uso del coche. Entre estas, sobresalen el género y la edad, ya que la primera está relacionada con la vida cotidiana (Hanson, 2010) y la segunda con el nivel socioeconómico y, por ende, con la posesión de automóvil 
(Schwanen et al., 2001), una de las características personales que más influyen en el modal choice.

\subsection{Movilidad a nodos de actividad suburbanos}

En las últimas décadas, las distancias y los tiempos del desplazamiento al lugar de trabajo o estudio se han alargado debido al crecimiento y la dispersión de las áreas metropolitanas. Se han construido entornos monofuncionales periféricos o suburbanos que ofrecen gran número de puestos de trabajo y, por tanto, generan numerosos desplazamientos cotidianos. De la separación territorial y funcional entre dichos nodos de actividad económica y la ciudad deriva una disminución de los desplazamientos activos y del uso de transporte público y un aumento del uso del coche privado. Unos hechos que han incrementado el interés académico por el análisis de estos entornos y que suponen un reto para las políticas públicas, cuando se quiere apostar por la movilidad sostenible. En este contexto, está apareciendo un buen número de trabajos centrados en las "universidades campus», como un ejemplo de entorno monofuncional (Gurrutxaga et al., 2017; Kotoula et al., 2017; Lavery et al., 2013). El caso de la UAB se enmarca en este contexto, con una peculiaridad destacable, la de tener una oferta de transporte público elevada con relación a su localización suburbana.

\section{Metodología}

\subsection{La fuente de información y método de análisis}

La encuesta Hábitos de Movilidad de la Comunidad Universitaria de la UAB $(\mathrm{UCMHS})^{1}$ es un trabajo estadístico sobre las características de los desplazamientos que realizan el colectivo estudiantil y el personal para llegar al campus universitario. Esta investigación se lleva a cabo desde el año 2001 de forma bianual. La edición de 2013 obtuvo una muestra de 5.814 respuestas validas, que significan el $12,5 \%$ del total de los miembros de la comunidad. Es una encuesta representativa con un margen de error del $\pm 1,29 \%$. La información que proporciona es de gran valor, no solo porque indica qué características tiene la movilidad cotidiana al trabajo o estudio de esta colectividad, sino también por el estudio longitudinal que representa. El cuestionario está estructurado en cuatro grandes bloques: (i) cuestiones generales sobre aspectos sociodemográficos de los participantes; (ii) hábitos de la movilidad cotidiana (iii) modos de transporte utilizados; y (iv) otras cuestiones.

El análisis descriptivo de la elección modal se ha realizado para toda la muestra y se ha estratificado según las características personales de los miembros de la comunidad. Los hábitos se compararon por rol y género mediante la

1. Más información en <http://www.uab.cat/web/la-mobilitat-a-la-uab/enquesta-de-mobilitat-1255501888126.html>. 
prueba de chi-cuadrado o t-test. Los análisis estadísticos se realizaron utilizando IBM SPSS v19 para Windows.

\subsection{El emplazamiento del campus de la $U A B$}

La UAB es una universidad campus aislada de cualquier trama urbana tradicional, de carácter suburbano, y localizada en la Región Metropolitana de Barcelona (RMB). Esta región tiene una superficie de $2.500 \mathrm{~km}^{2}$ y casi 5 millones de habitantes (2013), con un 63,4\% de población catalana y un 10,2\% española (Ajuntament de Barcelona, 2014). Se trata de un territorio con 164 municipios, cuyas dinámicas funcionales se estructuran bajo una lógica metropolitana, articulada a partir del municipio de Barcelona, en un radio de unos $40 \mathrm{~km}$. La UAB se ubica a $15 \mathrm{~km}$ de Barcelona, entre la primera corona metropolitana, conurbada al núcleo central, y una segunda, más alejada, estructurada a partir de diversas ciudades históricas de tamaño medio (Miralles-Guasch y Tulla Pujol, 2012). La ciudad central, Barcelona, con 1.602.386 habitantes, ha colmatado la urbanización del espacio edificable de su municipio y tiene unos niveles de densidad de población altos y homogéneos $\left(15.802,6\right.$ habitantes $\left./ \mathrm{km}^{2}\right)$. Más allá de Barcelona, las otras ciudades donde viven los miembros de la comunidad universitaria se hallan mayoritariamente en la segunda corona metropolitana y, aunque esta en su conjunto posee densidades muy inferiores a las de la capital, tienen — sobre todo las ciudades históricas - unas morfologías y funcionalidades muy similares a las de Barcelona (Pallares-Barbera et al., 2011).

El carácter suburbano del campus hace que casi la totalidad de los miembros de la comunidad requiera de transporte mecánico (privado o público) para llegar a la UAB, de manera que las infraestructuras ferroviarias y viarias tienen una gran importancia en la vida diaria de la universidad. Dos vías principales de gran capacidad, las autopistas AP-7 y C-58, se cruzan a muy poca distancia del campus. La AP-7, con tres puntos de acceso directos, enlaza la universidad con el resto de la Región Metropolitana. La C-58 también tiene conexión indirecta al campus por la vía local BV-1414. La red ferroviaria de cercanías tiene una notable presencia en la UAB, pues dos compañías (RENFE y FGC) le dan servicio, con tres estaciones dentro del recinto o en sus límites. Además, existen quince líneas de autobuses interurbanos que conectan el campus con ciudades próximas. Otro factor básico es la gratuidad del parking para toda la comunidad universitaria.

\subsection{La comunidad universitaria de la $U A B$}

En 2013 la comunidad universitaria de la UAB estaba formada por 47.866 personas, de las cuales el $86,6 \%$ eran estudiantes y $13,5 \%$ personal de universidad (profesores, investigadores y personal administrativo). El 58,5\% eran mujeres. Sin embargo, la distribución por género es distinta según el colectivo. Mientras que entre el colectivo estudiantil las mujeres representan el 60,5\%, en el de personal son el 51,9\%. La distribución por colectivos condiciona la 
Mapa 1. Universitat Autònoma de Barcelona en la Región Metropolitana de Barcelona

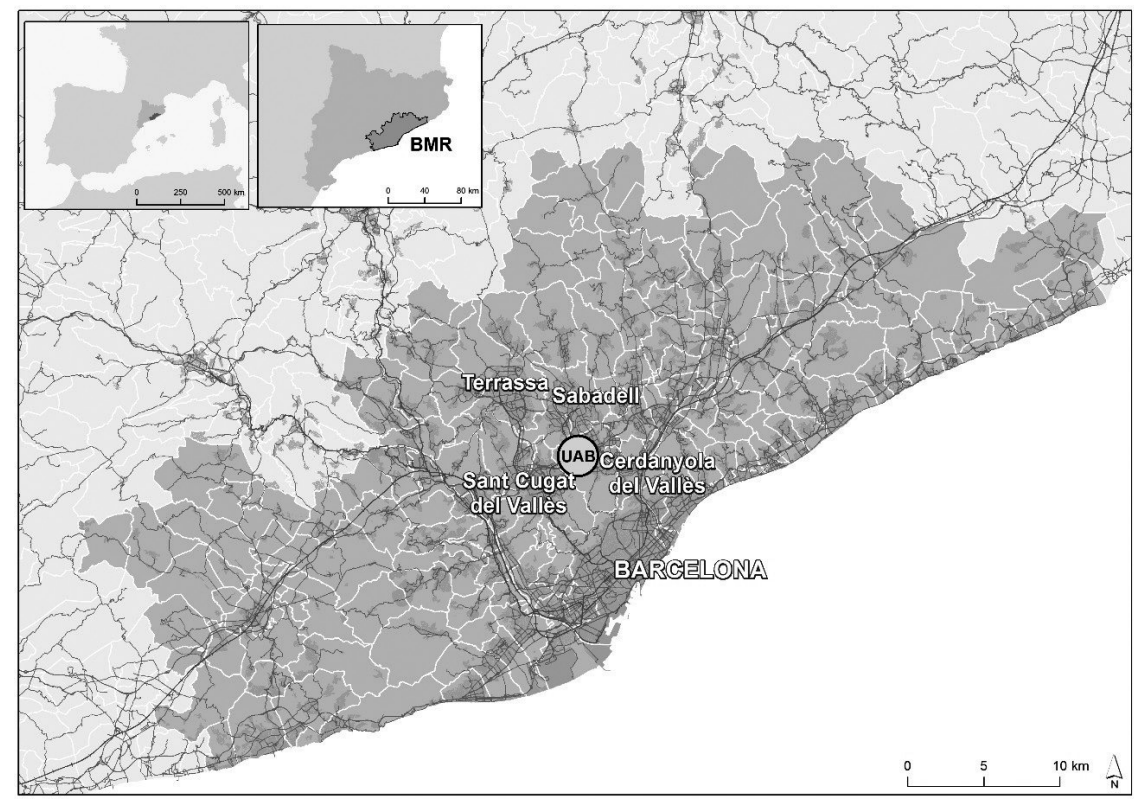

Fuente: Censo de 2011 (Instituto Nacional de Estadística).

edad media de la comunidad, que se sitúa en los 27 años y que aumenta con relación a los años de permanencia en la universidad. Así, mientras el conjunto de estudiantes tiene una media de 23,7 años, el personal se sitúa en los 43,5.

El 95\% de la comunidad universitaria vive en la Región Metropolitana de Barcelona, y el 59\% reside en uno de los cinco municipios metropolitanos alrededor de la universidad. Entre estos, destaca Barcelona, donde reside el $28 \%$, seguida de Sabadell (10\%), Cerdanyola del Vallès (10\%), Terrassa (7\%) y Sant Cugat del Vallès (3\%); un $1 \%$ vive en el propio campus (mapa 1 ). Todas estas ciudades, menos Terrassa, además de estar cerca del campus, tienen enlace directo mediante ferrocarril con el campus. Además, son ciudades con una morfología densa, compacta y de usos mixtos. La media de días que los miembros de la comunidad van a la $\mathrm{UAB}$ es de 4,4 a la semana.

\subsection{La comunidad universitaria}

Una de las características de la comunidad universitaria es su mayor nivel de homogeneidad, en comparación con el resto de la población. Muchos de los factores que la definen, como algunas particularidades de sus viajes, su entorno vital, cuestiones culturales o de estatus económico, son similares para el conjunto de sus miembros. Estas semejanzas realzan aquellos rasgos distintivos de 
los miembros de la universidad, ya que pueden analizarse aislándolos de los que les confieren más homogeneidad. Desde el punto de vista metodológico, es muy difícil encontrar un grupo de población con dichas similitudes sobre el cual disponer de información estadísticamente representativa de sus desplazamientos cotidianos. Por tanto, el análisis de una comunidad universitaria es un caso óptimo para poder tratar de forma aislada algunas variables que inciden en la elección modal. Las características que influyen en el modal choice (Witte et al., 2013) se dividen en cuatro grupos: las personas que viajan, el entorno donde se realizan los viajes, las características de los desplazamientos y sus hábitos. Cada uno de estos grupos está definido por distintas variables. En los miembros de la comunidad universitaria estas variables tienen muchas similitudes. Factores como el motivo, el destino, la necesidad del uso de un transporte motorizado, las condiciones climáticas o la información son similares para toda la comunidad. También los indicadores espaciales son muy homogéneos para el grupo estudiado, pues la densidad, la diversidad, la proximidad de las infraestructuras, la frecuencia del transporte público y la oferta de parking gratuito son similares para toda la población, ya que son características que ofrece, en general, la RMB o el propio campus universitario (GEMOTT, 2015). Al ser un viaje muy frecuente y repetitivo - los miembros de la comunidad van a la universidad una media de 4,4 días por semana-, también los aspectos sociopsicológicos son similares, pues sus experiencias, hábitos o percepciones son bastante homogéneos (Delclòs-Alió y Miralles-Guasch, 2017).

En cuanto a los niveles culturales y económicos, la comunidad universitaria difiere del resto de la población. El 43\% del alumnado de grado matriculado a primer curso $2014-2015$ en la UAB (el $45 \%$ por el total de universidades catalanas) se caracteriza por el nivel formativo familiar universitario (datos del Consejo Interuniversitario de Cataluña). Respecto al personal, compuesto por profesores, investigadores y personal administrativo, la mayor parte dispone de un título universitario. En cambio, solo el 25,6\% de la población de Cataluña, de 25 a 64 años, ha adquirido el tercer grado de educación (Idescat, 2011). El nivel de estudios está estrechamente relacionado con los ingresos. Según los datos del Idescat (2018), la renta media neta anual aumenta con el nivel de estudios: desde los $11.000 €$ correspondientes a personas con educación primaria hasta los $17.000 €$ de los que tienen educación superior. De todo ello, se puede deducir que la comunidad universitaria tiene niveles culturales y económicos más altos que el resto de la población.

Por otro lado, es necesario aislar las diferencias que pueden derivar del rol, estrechamente relacionado con la edad. Debido al cambio de recursos temporales relacionados con la carga familiar y el salto de estatus económico propio al momento de pasar de ser estudiante a tener un trabajo renumerado, el comportamiento del alumnado y del personal se trata de forma separada. Esta última variable está también relacionada con el tiempo de permanencia en la institución —el estudiantado permanece en la UAB una media de cinco años, mientras que el personal unos 25 años-, que puede influir en sus percepciones y sus hábitos. 


\section{Género y edad como factores que influyen en los desplazamientos en la UAB}

\subsection{Elección modal en los viajes laborales}

La comunidad universitaria utiliza mayoritariamente el transporte público para sus desplazamientos al campus $(59,8 \%)$. El 34,4\% usa los medios privados y solo un $5,8 \%$ va caminando o en bicicleta. El uso residual del modo activo tiene sus orígenes en la situación aislada y suburbana del campus de la UAB. Sin embargo, este reparto modal encubre diferencias significativas por colectivos y por géneros. El test chi-cuadrado confirma la influencia del colectivo en la elección modal $\left(X^{2}=33,319 ; p=0.001\right)$, más significativa para la comunidad estudiantil $\left(X^{2}=16,231 ; p=0.001\right)$ que para el personal $\left(X^{2}=9,443 ; p=0.01\right)$. Así, mientras más del $60 \%$ del alumnado utiliza el transporte público, este porcentaje es el que el personal de universidad recurre al automóvil. Pero las diferencias entre uno y otro colectivo, basadas en la edad y en los años de permanencia en la universidad, no son suficientes para entender la distribución modal de la comunidad universitaria. A ello se le tiene que añadir la variable género, ya que juega un papel fundamental en la elección modal. Entre el alumnado, las mujeres utilizan menos los transportes activos (una diferencia significativa de 3 puntos porcentuales) y son las mayores usuarias de los medios públicos (5,5 puntos de diferencia), en cambio emplean en menor medida el transporte privado. Tendencias que también aparecen en el personal de la universidad, pues aunque en este colectivo el uso del automóvil es mayoritario, ellas también lo utilizan menos (tabla 1).

Tabla 1. Elección modal de la comunidad universitaria de la UAB (\%)

\begin{tabular}{llcccc}
\hline Colectivo & & T. no motorizado & T. público & T. privado & Total \\
\hline 1. ${ }^{\text {er }}$ y $2 .^{\circ}$ ciclo & Hombres & $8,8^{\star \star}$ & $60,7^{\star}$ & 30,4 & 100 \\
& Mujeres & $5,8^{\star}$ & $66,2^{\star \star}$ & 28,0 & 100 \\
& Total & 7,0 & 63,9 & 29,1 & 100 \\
& & 100 & 100 & 100 & 100 \\
\hline PDI+PAS & Hombres & $5,0^{\star \star}$ & $32,7^{\star}$ & 62,4 & 100 \\
& Mujeres & $1,8^{\star}$ & $40,1^{\star \star}$ & 58,2 & 100 \\
& Total & 3,4 & 36,3 & 60,3 & 100 \\
& & 100 & 100 & 100,0 & 100 \\
\hline Total & Hombres & $7,2^{\star \star}$ & $55,8^{\star}$ & $37,1^{\star \star}$ & 100 \\
& Mujeres & $4,8^{\star}$ & $62,7^{\star \star}$ & $32,4^{\star}$ & 100 \\
& Total & 5,8 & 59,8 & 34,4 & 100 \\
& & 100 & 100 & 100 & 100 \\
\hline
\end{tabular}

\footnotetext{
* Valor significativamente bajo

${ }^{* *}$ Valor significativamente alto

Fuente: elaboración propia a partir de la encuesta UCMHS 2013.
} 


\subsection{Las condiciones de partida para el uso de los transportes privados: tener permiso de conducir y coche}

La mayoría de las personas de la comunidad universitaria de la UAB tienen permiso de conducir (70\%). Sin embargo, existen algunas diferencias entre géneros. Mientras que entre los hombres este porcentaje llega al 75\%, entre las mujeres se sitúa en el 66\%. No obstante, la obtención del permiso de conducir está directamente relacionada con la edad, por lo que, entre los dos colectivos estudiados, las diferencias son significativas. En el personal, alrededor del 90\% posee esta credencial, con unas diferencias mínimas entre géneros. En cambio, entre el estudiantado, su posesión es mucho menor (65\%), con diferencias de género notables: tiene el permiso un $62 \%$ de las estudiantes, mientras que entre sus homólogos masculinos esta cifra alcanza el 70\% (tabla 2).

Tener permiso de conducir es una condición necesaria, pero no suficiente, para poder utilizar un automóvil. Una segunda condición imprescindible es poseer coche y poder utilizarlo de forma cotidiana para ir al trabajo o al estudio. A esto hace referencia la tabla 3. El 54\% de los individuos de la comunidad universitaria declaran no tener un vehículo a su disposición para llegar a la UAB, una cifra que entre el alumnado llega al 60,5\% y entre el personal al $25 \%$. Existen diferencias de género significativas entre los más jóvenes, ya que el $63,5 \%$ de las alumnas manifiestan que no tienen coche, una cifra que baja al $56 \%$ entre los estudiantes.

Los datos que más explícitamente muestran la especificidad de la elección modal de la comunidad universitaria son los que cuantifican al colectivo que, a pesar de tener todos los requisitos para utilizar el transporte privado (carné de conducir + coche a su disposición), utiliza el público. El 34\% de los usuarios potenciales del transporte privado utilizan el público para llegar al campus. Un porcentaje que ha ido aumentando desde 2001 (primer año de la realización de la encuesta), cuando se situaba alrededor del 15\%. Unas cifras y una evolución

Tabla 2. Disposición del carné de conducir de la comunidad universitaria de la UAB (\%)

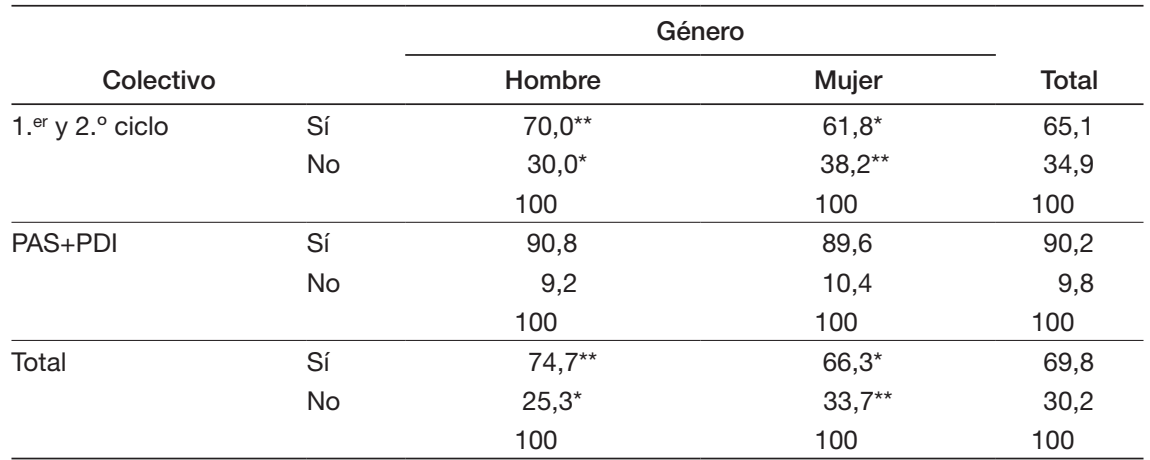

* Valor significativamente bajo

** Valor significativamente alto

Fuente: elaboración propia a partir de la encuesta UCMHS 2013. 
Tabla 3. Disposición de coche en la comunidad universitaria de la UAB (\%)

\begin{tabular}{|c|c|c|c|c|}
\hline \multirow[b]{2}{*}{ Colectivo } & & \multicolumn{2}{|c|}{ Género } & \multirow[b]{2}{*}{ Total } \\
\hline & & Hombre & Mujer & \\
\hline \multirow[t]{3}{*}{ 1. er y $2 .^{\circ}$ ciclo } & Sí & $44,0^{\star \star}$ & $36,5^{\star}$ & 39,5 \\
\hline & No & $56,0^{*}$ & $63,5^{\star \star}$ & 60,5 \\
\hline & & 100 & 100 & 100 \\
\hline \multirow[t]{3}{*}{ PAS+PDI } & Sí & 77,8 & 72,2 & 75,0 \\
\hline & No & 22,2 & 27,8 & 25,0 \\
\hline & & 100 & 100 & 100 \\
\hline \multirow[t]{3}{*}{ Total } & Sí & $51,6^{\star \star}$ & $42,3^{*}$ & 46,2 \\
\hline & No & $48,4^{*}$ & $57,7^{\star *}$ & 53,8 \\
\hline & & 100 & 100 & 100 \\
\hline
\end{tabular}

* Valor significativamente bajo

** Valor significativamente alto

Fuente: elaboración propia a partir de la encuesta UCMHS 2013.

que indican el buen nivel en la oferta de transporte público que tiene la universidad, pues en un entorno suburbano y en una localización aislada de cualquier trama urbana tradicional, en 2013, más de un tercio de los usuarios potenciales del transporte privado utilizan el público. Estas cifras indican el nivel de competitividad de este medio frente al automóvil. Sin embargo, dicho porcentaje es desigual por género y por edad. Mientras que para el alumnado se sitúa en el 33\%, entre el personal sube al 36\%. Lo más sorprendente es que, bajo las circunstancias antes mencionadas, los grupos que utilizan en menor medida el transporte privado son las mujeres del personal de la universidad (58\%) y los estudiantes hombres (60\%), colectivos que usan el público en un $40 \%$ frente al 33\% de sus homólogos masculinos, y el 35\% frente al 31\% de sus homólogas mujeres. Es decir, son los estudiantes hombres y las mujeres de más edad quienes, a pesar de tener todas las condiciones para utilizar el transporte privado, usan el público.

\subsection{Motivos para la elección modal según género}

Más allá de los medios de transporte que se utilizan, la encuesta indaga en las razones de su uso a través de una serie de preguntas cerradas donde se proponen posibles motivos de su elección. Aquí se han seleccionado las que hacen referencia al uso de los transportes mecánicos, privado y público, que son los mayoritarios.

La razón principal que declaran los entrevistados sobre su elección modal es el tiempo de viaje - este se identifica con dos de las respuestas que ofrece la encuesta: la rapidez y la proximidad al lugar de residencia, con unos porcentajes del 20,7\%, y del 16,5\%, respectivamente. Seguida de la oferta del transporte público $(15,4 \%)$ y del precio del viaje $(12,3 \%)$. Por lo tanto, para 
Tabla 4. Principales motivos de elección de un medio de transporte en la comunidad universitaria de la $U A B$, según género

\begin{tabular}{|c|c|c|c|}
\hline & \multicolumn{2}{|c|}{ Género } & \multirow[b]{2}{*}{ Total } \\
\hline & Hombre & Mujer & \\
\hline \multirow[t]{2}{*}{ Mejor combinación de horarios } & 46,2 & 53,8 & 100 \\
\hline & 11,1 & 9,5 & 10,2 \\
\hline \multirow[t]{2}{*}{ Más proximidad al lugar de residencia } & $35,9^{*}$ & $64,1^{\star \star}$ & 100 \\
\hline & 14,0 & 18,3 & 16,5 \\
\hline \multirow[t]{2}{*}{ Más barato } & $52,1^{\star \star}$ & $47,9^{\star}$ & 100 \\
\hline & 15,2 & 10,3 & 12,3 \\
\hline \multirow[t]{2}{*}{ Más rápido } & $47,7^{\star \star}$ & $52,3^{\star}$ & 100 \\
\hline & 23,4 & 18,8 & 20,7 \\
\hline \multirow[t]{2}{*}{ Más puntual o regular } & 42,4 & 57,6 & 100 \\
\hline & 5,8 & 5,8 & 5,8 \\
\hline \multirow[t]{2}{*}{ Más cómodo o agradable } & $53,3^{\star \star}$ & $46,7^{\star}$ & 100 \\
\hline & 11,0 & 7,1 & 8,7 \\
\hline \multirow{2}{*}{$\begin{array}{l}\text { Poca oferta de transporte público desde } \\
\text { el lugar de residencia u origen }\end{array}$} & $33,3^{*}$ & $66,7^{\star \star}$ & 100 \\
\hline & 12,2 & 17,8 & 15,4 \\
\hline \multirow[t]{2}{*}{ No tiene carné de conducir } & $22,4^{*}$ & $77,6^{\star *}$ & 100 \\
\hline & 2,3 & 5,7 & 4,3 \\
\hline \multirow[t]{2}{*}{ No dispone de vehículo privado } & 37,7 & 62,3 & 100 \\
\hline & 4,0 & 4,8 & 4,4 \\
\hline \multirow[t]{2}{*}{ No contesta } & $30,9^{*}$ & $69,1^{\star *}$ & 100 \\
\hline & 1,2 & 1,9 & 1,6 \\
\hline \multirow[t]{2}{*}{ Total } & 42,3 & 57,7 & 100 \\
\hline & 100 & 100 & 100 \\
\hline
\end{tabular}

* Valor significativamente bajo

** Valor significativamente alto

Fuente: elaboración propia a partir de la encuesta UCMHS 2013.

los miembros de la comunidad universitaria, más del $37 \%$ de las razones que se dan para elegir el medio de transporte están relacionadas con una mayor rapidez en el viaje y, en segundo lugar, con casi el $28 \%$, con la oferta del transporte público y el factor económico (tabla 4).

Sin embargo, si cada uno de los motivos de elección de un medio se contrasta por el género, existen algunas diferencias interesantes que no se centran solo en el tiempo, sino también en la calidad del viaje (más cómodo o agradable) y en la oferta de transporte público. Mientras que el primer motivo - rapidez- viene señalado por un 23,4\% de los hombres, las mujeres lo hacen en un $18,8 \%$. En segundo y tercer lugar, los hombres indican la importancia del precio $(15,2 \%)$ y de la proximidad al lugar de residencia $(14 \%)$. Esta última razón fue la que indican las mujeres en segundo lugar $(18,3 \%)$, mientras que en tercer lugar ellas señalan la oferta del transporte público $(17,8 \%)$. En resumen, existen algunas diferencias de género en lo 
referente al tiempo, ya que los hombres lo subrayan en mayor medida que las mujeres, así como el precio y la comodidad. Las mujeres, en cambio, destacan la proximidad al lugar de residencia, la oferta de transporte en general y también no disponer de carné de conducir como factores condicionantes de su elección.

\subsection{Motivos para la elección modal según edad y género}

Sin embargo, para entender bien las diferencias de motivos expresados según el género, se tiene que introducir la edad, pues las respuestas de hombres y mujeres pueden ser muy distintas según el colectivo (estudiantes o personal) del que forman parte (tabla 5).

Así, indagando en la edad con relación al porqué se utiliza el transporte privado, se observa que en edades más avanzadas la combinación de horarios y la rapidez son más importantes; con una diferencia de más de 10 puntos entre el estudiantado y el personal. En cambio, para los más jóvenes, la comodidad (el 13\% respecto al 10\%) y la poca oferta de transporte público (el $22 \%$ respecto al $19 \%$ ) son razones más importantes, aunque con diferencias menores, respecto a las razones apuntadas mayoritariamente por el personal.

Tabla 5. Motivos del uso de coche en la comunidad universitaria de la UAB (\%)

\begin{tabular}{|c|c|c|c|c|c|c|}
\hline \multirow[b]{2}{*}{ Motivos } & \multicolumn{3}{|c|}{ Estudiantes } & \multicolumn{3}{|c|}{ PAS+PDI } \\
\hline & Hombre & Mujer & Total & Hombre & Mujer & Total \\
\hline \multirow[t]{2}{*}{ Mejor combinación de horarios } & 36,7 & 63,3 & 100 & 48,6 & 51,4 & 100 \\
\hline & 8,6 & 10,9 & 9,9 & 14,0 & 16,2 & 15,1 \\
\hline \multirow[t]{2}{*}{ Más proximidad al lugar de residencia } & 35,7 & 64,3 & 100 & 60,0 & 40,0 & 100 \\
\hline & 1,2 & 1,6 & 1,4 & 2,4 & 1,8 & 2,1 \\
\hline \multirow[t]{2}{*}{ Más barato } & $55,6^{\star *}$ & $44,4^{*}$ & 100 & 50,0 & 50,0 & 100 \\
\hline & 10,7 & 6,3 & 8,2 & 1,6 & 1,8 & 1,7 \\
\hline \multirow[t]{2}{*}{ Más rápido } & 43,1 & 56,9 & 100 & 53,8 & 46,2 & 100 \\
\hline & 41,4 & 40,5 & 40,9 & 51,2 & 48,2 & 49,8 \\
\hline \multirow[t]{2}{*}{ Más puntual o regular } & 36,1 & 63,9 & 100 & 66,7 & 33,3 & 100 \\
\hline & 3,1 & 4,0 & 3,6 & 3,2 & 1,8 & 2,5 \\
\hline \multirow[t]{2}{*}{ Más cómodo o agradable } & $53,8^{\star \star}$ & $46,2^{*}$ & 100 & 55,3 & 44,7 & 100 \\
\hline & 16,7 & 10,6 & 13,2 & 10,4 & 9,2 & 9,8 \\
\hline \multirow{2}{*}{$\begin{array}{l}\text { Poca oferta de transporte público } \\
\text { desde el lugar de residencia u origen }\end{array}$} & $34,3^{*}$ & $65,7^{\star \star}$ & 100 & 47,2 & 52,8 & 100 \\
\hline & 17,6 & 25,0 & 21,9 & 16,8 & 20,6 & 18,6 \\
\hline \multirow[t]{2}{*}{ No contesta } & 33,3 & 66,7 & 100 & 50,0 & 50,0 & 100 \\
\hline & 0,7 & 1,1 & 0,9 & 0,4 & 0,4 & 0,4 \\
\hline \multirow[t]{2}{*}{ Total } & 42,5 & 57,5 & 100 & 52,3 & 47,7 & 100 \\
\hline & 100 & 100 & 100 & 100 & 100 & 100 \\
\hline
\end{tabular}

* Valor significativamente bajo

${ }^{* *}$ Valor significativamente alto

Fuente: elaboración propia a partir de la encuesta UCMHS 2013. 
Si a estas diferencias se le añade el género, entre el alumnado las respuestas son más diversas que entre el personal. Así, entre la juventud, el precio $(11 \%$ vs. $6 \%)$, y la comodidad ( $17 \%$ vs. $11 \%$ ) son más importantes para los hombres que para las mujeres. En cambio, ellas hacen más hincapié en la poca oferta de transporte público $(18 \%$ vs. $25 \%)$ y en la combinación de horarios $(9 \%$ vs. $11 \%$ ). Estas diferencias también se observan en el personal de la universidad, donde las mujeres mayores subrayan más que los hombres estas mismas razones. Sin embargo, a pesar de que las diferencias de género tienen lugares comunes, las intensidades son menos pronunciadas en las edades mayores que entre los jóvenes.

Por lo que se refiere al transporte público, las grandes diferencias entre los distintos colectivos se encuentran en la comodidad, con una discrepancia de más de 11 puntos a favor del personal de la universidad (16\% vs. 4\%), y los motivos relacionados con la posibilidad de utilización del vehículo privado, priorizados por los y las estudiantes en mayor medida que por el personal: solo el $1 \%$ de los mayores señala el hecho de no tener carné de conducir como motivo de utilización del transporte público, en cambio para el $8 \%$ del colectivo estudiantil es importante. También la oferta de transporte público es más significativa para los jóvenes (15\%) que para los mayores $(5 \%)$.

Si a la edad se le añade el género, donde se perciben más diferencias es entre el alumnado. Por orden de importancia, los motivos a los que las mujeres estudiantes dan mayor relieve son la posesión del carné de conducir ( $10 \%$ vs. $4 \%)$, la oferta de transporte público (17\% vs. $11 \%)$ y una oferta próxima al lugar de residencia (26\% vs. $22 \%)$. En cambio, los hombres estudiantes subrayan el precio ( $19 \%$ vs. $12 \%)$, la rapidez $(10,3 \%$ vs. $6,4 \%)$, la comodidad $(6 \%$ vs. $3 \%)$ y la combinación de horarios $(12 \%$ vs. $8 \%)$. En el personal, aunque las diferencias no son tan acentuadas entre géneros, los motivos priorizados por los hombres y por las mujeres son iguales a los de los más jóvenes.

\section{Conclusiones}

La literatura académica ubica las razones de uso de los distintos medios de transporte entre dos coordenadas: las condiciones del territorio y las características individuales o colectivas de las personas que se desplazan. En el eje territorial, la localización central o periférica en las áreas metropolitanas, sus características morfológicas, su diversidad de usos y el nivel de oferta de transporte público son razones fundamentales para utilizar uno u otro medio de transporte. En el eje personal, el género y la edad son características esenciales, ya que, además de tener un papel influyente en sí mismas, condicionan a otros factores, como la disposición de carné de conducir y de coche, el nivel de ingresos y la estructura del hogar, entre otros. Estas son las características que determinan el modal choice de cada individuo y que configuran el modal split de una colectividad. 
Así pues, el reparto modal de una comunidad es multifactorial, lo que confiere al análisis un nivel alto de complejidad e incluso de confusión. En este sentido, es importante poder estudiar colectivos con algunos factores similares que faciliten el análisis de las diferencias en otros factores. En este caso, el colectivo bajo estudio comparte un mismo entorno de trabajo, unas mismas características de viaje, así como perfiles socioeconómicos altamente similares. Una situación excelente para dilucidar la influencia de otros factores, como la edad y el género, en la distribución modal. Estas dos variables se apuntan en la literatura académica como fundamentales para entender tanto el uso como el acceso a los distintos medios de transporte (Witte et al., 2013; Marquet y Miralles-Guasch, 2014; Schwanen et al., 2001). En este estudio, el reparto modal es una cuestión de edad, en la medida en que, con los años y con el incremento del tiempo de permanencia en la institución académica, se utiliza en mayor proporción el transporte privado (60\%). Sin embargo, si se focaliza en las personas que, a pesar de tener todas las condiciones para utilizar el coche (carné y coche a disposición), usan el transporte público, empezamos a considerar algunas diferencias de género, pues las mujeres son las que, con la edad, abandonan el transporte privado y se pasan al público. En cambio, en los hombres esta situación es la contraria: los hombres estudiantes, más jóvenes, a pesar de tener todas las posibilidades de utilizar el coche, usan el transporte público. La edad aquí, en relación con el género, tiene un efecto opuesto. En este sentido, se tendría que profundizar en elementos que afecten más directamente a las mujeres jóvenes, como por ejemplo la percepción de la inseguridad personal, ya que esta suele estar sobreestimada por ellas en relación al riesgo real (Oberhauser et al., 2017).

Si más allá de la descripción numérica nos adentramos en las razones que unos y otros expresan, el motivo prioritario para el uso del automóvil es el tiempo de viaje, que se expresa de distintas formas en casi un $60 \%$ de las respuestas. Es un motivo recurrente en muchos de los estudios sobre esta cuestión (Miralles-Guasch et al., 2014). Sin embargo, en lo que se refiere a la elección de un modo de transporte, la diferencia por género señala que los motivos más subjetivos entre hombres y mujeres son bastante distintos. Mientras ellos señalan el tiempo, el precio y la comodidad, ellas apuestan por la oferta de transporte público cercana al lugar de residencia y por el hecho de no disponer de carné de conducir.

Son especialmente interesantes las respuestas dadas por las mujeres que parecen situarse como usuarias cautivas del transporte público. Esta dependencia aparece tanto de forma directa, al declarar que no disponen de carné de conducir, como de forma indirecta, al priorizar la proximidad y, por tanto, la rapidez y la facilidad de acceso al transporte público. Este vínculo entre el colectivo femenino y la importancia de tener acceso a la red del transporte público en la proximidad coincide con los hallazgos de Marquet y MirallesGuasch (2014) sobre localización de los espacios de actividad de las mujeres en la escala local. Estas diferencias de género se pueden matizar cuando se trata del colectivo joven o del grupo más sénior. 
Este artículo avanza en el conocimiento sobre cómo los distintos perfiles personales influyen en el uso de los medios de transporte. Esta información es fundamental para que el diseño y la implementación de políticas dirigidas a la reducción del uso del automóvil sean eficaces (Burian et al., 2018). El caso de la UAB puede ser similar a otros nodos de actividad económica metropolitanos y suburbanos que generan numerosos desplazamientos. Las conclusiones pueden ser relevantes para la gestión de movilidad en otros campus universitarios, polígonos industriales o empresariales.

\section{Referencias bibliográficas}

Adams, J. (1999). The social implications of hypermobility. París.

Ademe (2009). «Energy Efficiency Trends and Policies in the Transport Sector in the EU | ODYSSEE-MURE project».

Ajuntament de Barcelona. Barcelona en xifres 2013 (2014). Recuperat de <https:// bcnroc.ajuntament.barcelona.cat/jspui/bitstream/11703/91979/1/13097.pdf>.

Banister, D. (2011). "Cities, mobility and climate change». Journal of Transport Geography, 19, 1.538-1.546. $<$ http://doi.org/10.1016/j.jtrangeo.2011.03.009>

Внат, C. R. (1998). "Analysis of travel mode and departure time choice for urban shopping trips». Transportation Research Part B: Methodological, 32 (6), 361-371.

BLACK, W. R. (2010). Sustainable transportation: problems and solutions. Nueva York: Guilford Press.

Burian, J.; ZajíčKovÁ, L.; Ivan, I. y MackŮ, K. (2018). «Attitudes and Motivation to Use Public or Individual Transport: A Case Study of Two Middle-Sized Cities». Social Sciences, 7 (6), 83. <http://doi.org/10.3390/socsci7060083>

Cerin, E.; Leslie, E.; Toit, L. du; Owen, N. y Frank, L. D. (2007). «Destinations that matter: Associations with walking for transport». Health \& Place, 13, 713-724. $<$ http://doi.org/10.1016/j.healthplace.2006.11.002>

Ciuffini, F. M. (1993). «El sistema urbà i la mobilitat horitzontal de persones, matèria i energia» ("The Urban System and the Horizontal Mobility for People, Matter and Energy»). Medi Ambient. Tecnologia i Cultura, 5, 42-53.

Delclòs-Alió, X. y Miralles-Guasch, C. (2017). «Suburban travelers pressed for time: Exploring the temporal implications of metropolitan commuting in Barcelona». Journal of Transport Geography (octubre), 0-1. $<$ http://doi.org/10.1016/j.jtrangeo.2017.10.016>

Ewing, R. y Cervero, R. (2002). «Travel and the Built Environment A Meta-Analysis». Journal of American Planning Association, 76 (3), 265-294. $<$ http://doi.org/10.1080/01944361003766766>

Garcia-Ramon, M. D.; Ortiz, A. y Prats, M. (2004). «Urban planning, gender and the use of public space in a peripherial neighbourhood of Barcelona». Cities, 21 (3), 215-223. $<$ http://doi.org/10.1016/j.cities.2004.03.006>

Garcia-Sierra, M.; Bergh, J. C. J. M. van den y Miralles-Guasch, C. (2015). «Behavioural economics, travel behaviour and environmental-transport policy». Transportation Research Part D: Transport and Environment, 41, 288-305. $<$ http://doi.org/10.1016/j.trd.2015.09.023> 
GEMOTT (Grup d'Estudis en Mobilitat Transport i Territori) (2015). Enquesta d'Hàbits de Mobilitat de la comunitat universitària de la UAB 2015. Universitat Autònoma de Barcelona.

Gurrutxaga, I.; Iturrate, M., Oses, U. y Garcia, H. (2017). «Analysis of the modal choice of transport at the case of university: Case of University of the Basque Country of San Sebastian». Transportation Research Part A: Policy and Practice, 105, 233-244. <http://doi.org/10.1016/j.tra.2017.04.003>

Gutiérrez, J. y García-Palomares, J. C. (2007). «New spatial patterns of mobility within the metropolitan area of Madrid: Towards more complex and dispersed flow networks». Journal of Transport Geography, 15, 18-30. <http://doi.org/10.1016/j.jtrangeo.2006.01.002>

Hanson, S. (2010). «Gender and mobility: new approaches for informing sustainability». Gender, Place \& Culture, 17 (1), 5-23. <https://doi.org/10.1080/09663690903498225>

Hickman, R.; Hall, P. y Banister, D. (2013). «Planning more for sustainable mobility». Journal of Transport Geography, 33, 210-219.

IDESCAT - Institut d'Estadística de Catalunya (2011). Nivell d'instrucció de la població de 16 anys i més. Per sexe i grups d'edat. Recuperat de <https://www.idescat.cat/ indicadors/?id=anuals\&n=10368\&tema=educa $>$.

- (2018). Renda mitjana neta anual per persona i unitat de consum per nivell de formació assolit (16 anys i més). Recuperat de <https://www.idescat.cat/ pub/?id=ecv\&n=7601>.

Kotoula, K. M.; Sialdas, A.; Botzoris, G.; Chaniotakis, E. y Salanova Grau, J. M. (2017). "Exploring the Effects of University Campus Decentralization to Students' Mode Choice». Periodica Polytechnica Transportation Engineering, 46 (4), 207-214. <http://doi.org/10.3311/PPtr.11641>

Lavery, T. A.; PÁez, A. y Kanaroglou, P. S. (2013). "Driving out of choices: An investigation of transport modality in a university sample». Transportation Research Part A, 57, 37-46. <http://doi.org/10.1016/j.tra.2013.09.010>

LAw, R. (1999). "Beyond "women and transport": towards new geographies of gender and daily mobility. Progress in Human Geography, 23 (4), 567-588. <http://doi.org/10.1191/030913299666161864>

Limtanakool, N.; Dijst, M. y Schwanen, T. (2006). «The influence of socioeconomic characteristics, land use and travel time considerations on mode choice for medium- and longer-distance trips». Journal of Transport Geography, 14 (5), 327-341. <http://doi.org/10.1016/j.jtrangeo.2005.06.004>

Litman, T. (2012). Land Use Impacts on Transport: How Land Use Factors Affect Travel Behaviour. Canadá: Victoria Transport Policy Institute.

LundBerg, B. y Weber, J. (2014). «Non-motorized transport and university populations: an analysis of connectivity and network perceptions». Journal of Transport Geography, 39, 165-178. <http://doi.org/10.1016/j.jtrangeo.2014.07.002>

Marquet, O. y Miralles-Guasch, C. (2014). "Walking short distances. The socioeconomic drivers for the use of proximity in everyday mobility in Barcelona». Transportation Research Part A: Policy and Practice, 70, 210-222. <http://doi.org/10.1016/j.tra.2014.10.007> 
Miralles-Guasch, C. (2002). Ciudad y Transporte: El Binomio Imperfecto (City and Transportation. The Imperfect Binomial). Barcelona: Arial.

- (2012). "Las encuestas de movilidad y los referentes ambientales de los transportes». EURE, 38 (115), 33-45.

Miralles-Guasch, C.; Martínez-Melo, M. y Marquet, O. (2014). «On user perception of private transport in Barcelona Metropolitan area: an experience in an academic suburban space». Journal of Transport Geography, 36, 24-31. <http://doi.org/10.1016/j.jtrangeo.2014.02.009>

Miralles-Guasch, C.; Melo, M. M. y Marquet, O. (2016). «A gender analysis of everyday mobility in urban and rural territories: from challenges to sustainability». Gender, Place \& Culture, 23 (3), 398-417. <http://doi.org/10.1080/0966369X.2015.1013448>

Miralles-Guasch, C. y Tulla Pujol, A. F. (2012). «La región metropolitana de Barcelona. Dinámicas territoriales recientes». Boletín de la Asociación de Geógrafos Españoles, 58, 299-318.

NÆss, P. (2015). «Built Environment, Causality and Travel». Transport Reviews, 35 (3), $1-17$. <http://doi.org/10.1080/01441647.2015.1017751>

Oberhauser, A.; Fluri, J. L.; Whitson, R. y Mollett, S. (2017). Feminist Spaces: Gender and Geography in a Global Context. Nueva York: Routledge.

OrY, D. T. y Mokntarian, P. L. (2009). «Modeling the structural relationships among short-distance travel amounts, perceptions, affections, and desires». Transportation Research Part A: Policy and Practice, 43 (1), 26-43. <http://doi.org/10.1016/j.tra.2008.06.004>

Pallares-Barbera, M.; Badia, A. y Duch, J. (2011). "Cerdà and Barcelona: The need for a new city and service provision Cerdà and Barcelona: The need for a new city and service provision». Urbani Izziv, 22 (2), 122-136. <http://doi.org/10.5379/urbani-izziv-en-2011-22-02-005>

Рiтомво, C. S.; Kawamoto, E.; Sousa, A. J. (2011). «An exploratory analysis of relationships between socioeconomic, land use, activity participation variables and travel patterns». Transport Policy, 18 (2), 347-357. <http://doi.org/10.1016/j.tranpol.2010.10.010>

Rodrigue, J. P.; Comtois, C. y SLACK, B. (2009). The Geography of Transport Systems (segunda ed.). Londres: Routledge.

Scheiner, J. (2010). "Interrelations between travel mode choice and trip distance: trends in Germany 1976-2002». Journal of Transport Geography, 18(1), 75-84. <http://doi.org/10.1016/j.jtrangeo.2009.01.001>

Schwanen, T.; Banister, D. y AnAble, J. (2011). «Scientific research about climate change mitigation in transport: A critical review». Transportation Research Part A: Policy and Practice, 45 (10), 993-1006. <http://doi.org/10.1016/j.tra.2011.09.005>

Schwanen, T.; Dijst, M. y Dieleman, F. M. (2001). «Leisure trips of senior citizens: determinants of modal choice». Tijdschrift Voor Economische En Sociale Geografie, 92 (3), 347-360. <http://doi.org/10.1111/1467-9663.00161>

Tiwari, R.; Cervero, R. y Schipper, L. (2011). «Driving CO reduction by Integrating Transport and Urban Design strategies». Cities, 28 (5), 394-405. <http://doi.org/10.1016/j.cities.2011.05.005> 
Witte, A. de; Hollevoet, J.; Dobruszkes, F.; Hubert, M. y Macharis, C. (2013). «Linking modal choice to motility: A comprehensive review». Transportation Research Part A: Policy and Practice, 49, 329-341. <http://doi.org/10.1016/j.tra.2013.01.009>

Woodcock, J.; Banister, D. y Edwards, P. (2007). «Energy and transport». The Lancet, 370, 1.078-1.088.

$<$ http://doi.org/10.1016/S0140-6736(07)61254-9> 